\title{
Why Does Tea' Stone Have the Potential to Succeed? Based on the POCD Analysis Framework
}

\begin{abstract}
Haoqi $\mathrm{Xiao}^{1, *}$
${ }^{1}$ Smeal College of Business, Pennsylvania State University

*Email: hvx5048@psu.edu

ABSTRACT

This paper analyses a new emerging pure tea brand, compared with many tea-related beverage stores that appear in the market. According to the research, people's acceptance of such products is higher and higher, and the market competition is also intensified. The main purpose is to explain to readers why tea' stone, an emerging and innovative pure tea beverage brand, has the potential to succeed in an increasingly competitive market. However, as a new brand of pure tea, Tea' stone has many shortcomings. It faces many difficulties and challenges, such as slow development and low reputation. This article presents a comprehensive review of Tea' stone and analyses why it has the potential of success from many aspects. The paper mainly uses the POCD framework to analyze tea' stone. This model can well analyze the competitive advantages or shortcomings of a brand from many aspects. In conclusion, based on the model's analysis and survey results, it suggests that Tea' stone is very competitive in the current market and can be expected in the future.
\end{abstract}

Keywords: Tea' Stone, POCD analysis, tea-related beverage stores.

\section{INTRODUCTION}

Tea' stone was founded in 2018 and mainly sells pure tea drinks. It sells many kinds of pure tea drinks, tea' stone operates its pure tea shop by using the bubble tea shop model. Its special point is to provide customers with a quiet and dedicated place to socialize and drink tea. Tea' stone team believes that pure tea drinks will not disappear due to social progress but need more contemporary expression to adapt to the changing needs of consumer groups. To better analyze this start-up enterprise, this paper needs to comprehensively study the potential, advantages, and disadvantages from multiple directions. However, the general analysis method does not apply to this company because the nature of this company is different from that of ordinary listed or unlisted companies. This company has just experienced several preliminary rounds of financing and is still in the preliminary development stage. Therefore, there is no transparent financial report, and there is not enough information in the market. Therefore, this paper will use the POCD framework to slowly analyze the market potential of this company.

Before start analyzing, the article will introduce the POCD framework first. According to Manish Chaulagain (May 9, 2020) [1], as an entrepreneur, who is looking to grab the opportunity, it is most productive to look at it as an interconnected combination of four elements: people, context, (business) opportunity, and deal. The right combination, i.e. the degree of fit among them, means a high-potential opportunity. A bad combination, or the lack of any single element, is a recipe for failure. Since the company to be analyzed in this article has not been listed and is in an initial stage of development. There is almost no transparent transaction information, this article will only use people, opportunity and context these three parts for analysis. Manish Chaulagain's article explains the specific meaning of this paragraph and the three parts. On the people side, it is the people who make a huge impact on a company, regardless of whether they are actual employees. These can be founders or investors, key suppliers, and in some cases, customers. On the opportunity side, it is a contemplated product or service for which customers are willing to pay more than the required investment and operating costs, in the part of the context. These factors affect the outcome of the opportunity but are generally beyond the direct control of management. This category includes interest rates, regulations, macroeconomic activities, technology, and some industry variables such as competition or the relative bargaining power of companies [2]. On this basis, we conclude that each part of the POCD framework plays an important analytical role, and they complement each other. Using the POCD framework to analyze tea' stone, 
a start-up company can better know its competitive potential.

\section{ANALYSIS OF TEA' STONE- POCD FRAMEWORK}

\subsection{People}

Tea' stone is a company with great development potential, which is mainly based on three aspects: human, opportunity and environmental factors. Firstly, Tea' stone has certain advantages at the founder level. The founder, Li Shu, is graduated from the Academy of Arts \& Design, Tsinghua University. Also, she is the strong stockholder of the second-biggest shareholder of tea' stone: Shenzhen Tea' stone Investment Partnership. This is actually a good advantage because many successful founders have high academic qualifications. In China, they graduated from the Department of art and design of Tsinghua University, which laid a good foundation for developing Tea' stone. Based on the brand concept of tea' stone, it is an innovative pure tea brand. The educational background of the founders can help the growth of the enterprise. The chief product officer, Gu Zhuoheng, also plays an important role in product design and the overall architecture design. $\mathrm{He}$ is graduated from the Communication University of China, and he is a professional broadcast host. At the same time, he has many years of research experience in tea culture and the tea industry. So, he knows how to improve the customer's experience of the product.

The founding team is important too. By using Zhaona's article (March 31, 2021) [3], it can be understood that the Founding team participated in the creative planning and brand communication of largescale cultural tourism projects in the OCT Cultural Industry platform and provided brand consulting services for several benchmark commercial complexes in large cities in China. In the view of the tea' stone team, tea has a way of expression in line with the live scene at that time in every era. At present, what young people refuse is not the tea itself, but the way of drinking tea that is not suitable for their lifestyle. Tea' stone's solution is a "new retail model of immersive experience" with pure tea consumption as the core. The key to its innovation is to solve the pain points of tea consumption in the market and let young consumers have a strong interest in tea culture and are willing to experience and actively spread it. On this basis, the combination of the cultural value of Chinese tea and the theoretical system of modern aesthetic design, Tea' stone carries out an innovative design of Chinese tea based on the drinking method, the apparatus, the packaging, the environment, the experience and Marketing. Experiences, packaging, environments and even furniture are originally designed by the team and then optimized and iterated in combination with market needs and consumer scenes.
The launch of the new product series is combined with a complete tea-drinking lifestyle set, from brewing mode to device presentation to retail tea packaging, all of which have been designed and consistently polished with a professional aesthetic in mind [4].

In Zhangting's article, she thinks that customers can not only feel the tea culture but also drink "a pot of good tea" in a simple way and are willing to take photos and share. In addition to in-store consumption, tea' stone's tea products can also be purchased at retail, and the touching scene extends from in-store to consumers' family scene. The tea' stone team has experience in both aesthetic design and business logic. $80 \%$ of the team members are art and design majors and have established their own innovation center. At the same time, the team has also studied tea and tea culture for more than ten years, understood modern beverage culture and scientific and technological tea drinking methods, and is good at innovation in visual art, commercial aesthetics, spatial creativity, etc. The team relies on modern design language and traditional culture to empower brands and products and provide consumers with innovative experiences [5].

On this basis, the experience of the team is very important. Based on Zhangting's opinion, it is understood that the founding team of tea' stone has worked together for more than ten years. Before starting the business, it participated in the creative planning and brand communication of large-scale cultural and tourism projects in Oct on the cultural industry platform of OCT. It provided brand consulting services for benchmark commercial complexes in several large cities in China. Founder Li Shu graduated from the Academy of fine arts of Tsinghua University; Gu Zhuoheng, chief product officer, graduated from the Communication University of China. He is a professional broadcasting host. At the same time, he has many years of research experience in the field of tea culture and the tea industry [6].

\subsection{Opportunity}

\subsubsection{Based on market information}

Tea' stone is using a very creative way to operate its pure tea store, people who go into the store will find this way is very novel and would like to try it, the product of the Tea' stone is also attractive enough for customers to go back and have it try again. Tes' stone provides the customer with high quality and comfortable place for the customer to enjoy tea. Tea' stone saves customers the troublesome steps of making tea. Customers only need to choose the tea they want on the menu, and a pot of brewed tea will be delivered to them every soon, making customers completely enjoy the relaxation moment. In my opinion, Tea occupies a very important position in Chinese culture. Many people who love tea will easily become loyal customers of Tea' stone because the tea 
lovers can find everything they want in the Tea' stone store. It is worth mentioning that two strong and experienced venture capital companies are very optimistic about Tea' stone's progress and have invested tens of millions of RMB in Tea' stone. I think this is a good signal to Tea' stone's future development. Lastly, Tea' stone is the only company in this special pure tea market, it has no direct competitors, so there is no great pressure to survive for Tea' stone.

The brand has opened four Direct Stores in Shenzhen with each single store area of 200-400 square meters. The store in Vientiane Tiandi has been open for three months and has monthly sales of nearly 1.5 million yuan, including retail revenue from take-out accounts for $30 \%$. Currently, the tea stone single-store model is polished and mature. In 2021 it will accelerate the expansion of shops and penetrate the central business circle of other cities of the first category. The high-turnover business model with the high gross profit Increasingly standardized margins and supply chain systems have developed the tea market rapidly in recent years. Hence, the emergence of new tea beverages, but the focus is not on the tea itself. Tea and fruit tea. Tea only plays the background role of tea, not the main selling point. With the development of tea products from pearl milk tea to dessert, ingredients such as milk lid, fairy grass, soy milk and cassava will continue to weaken the tea's sense of existence even more and more. Drinking tea has been a Chinese habit for thousands of years. It's also an addicting category like tobacco, wine, coffee, and sugar. It has a sufficiently large market around the world. The Tea' stone team believes that pure tea is drunk alone. It will not go away with social progress, but it needs a more contemporary expression to adapt to the changing needs of consumer groups [7].

According to Huyue, general manager of Tea' stone, told $36 \mathrm{kr}$, the answer is to have beauty, fun and materials to provide young people with fashionable ways to drink tea. For many young people, drinking pure tea is an "oldfashioned" behavior. Even in many people's impression, the taste of pure tea is bitter and astringent. The reason lies in the lack of innovation in the production, brewing and presentation of Chinese tea for a long time. In fact, China is rich in tea products, and many teas have a unique flavor, which is worthy of in-depth exploration. Tea' stone's innovation is to change the way young people drink tea. Specifically, in the selection stage, the team established a set of selection criteria, directly selected from the major tea originated in China, and then selected 108 types of tea from tens of thousands of products to present to consumers, covering yellow tea, green tea, white tea, oolong tea, black tea, black tea, flower tea and other categories. With good tea, how to brew is the part that most young people are not good at, but the form of special service in traditional teahouses is too complex and easy to make young consumers feel "uncomfortable". Tea' stone provides a "one for one" service. After the customer places an order, the tea artist completes the brewing at the bar in the store and provides the brewed tea soup to consumers. While retaining the sense of ceremony, it provides an appropriate "tea atmosphere" for young people. Based on 108 types of tea, tea' stone innovated around the new playing and drinking methods of pure tea from traditional tea cooking to modern technology machine extraction and built a new product system and experience based on technological innovation [8].

However, the tea' stone has some weaknesses. The Tea' stone was found in 2018, 3 years have passed, it only opened 5 stores with a huge amount of financial support, which is a relatively slow growth compared to other companies. Also, Tea' stone did not do a good job on propaganda, because other than tea lovers and pure tea drinkers, not that many people have heard about Tea' stone. This is relatively weak propaganda compared to other companies as well. Because Tea' stone is operated in the mode of opening physical stores. At the same time, these stores are opened in the center of large shopping malls or very prosperous areas. Therefore, the rental cost of the store is very expensive. Even with tens of millions of financial supports, the cost of maintaining and opening a new store is also a great expenditure. Lastly, even though tea is important in Chinese culture, not every Chinese person likes to drink tea. Therefore, in this case, the market share of tea lovers becomes very important. This is related to the upper limit of the scale of Tea' stone, but because the scale of Tea' stone is still very small, it is difficult to predict the future of this market.

\subsubsection{Based on a survey-collected data}

At present, because Tea' stone is still in its infancy, there are only four stores in Shenzhen, which leads to the low popularity of the brand. At the same time, Tea' stone did not choose to vigorously publicize in the early stage of development, and the development model of physical stores requires a long-time cycle, so the growth of Tea' stone is relatively slow at this stage. Correspondingly, Tea' stone has a certain popularity in the circles of the younger generation who love tea culture. Most of these people are concentrated near physical stores. The market research results suggest that a large proportion of young people are willing to try this popular and convenient tea shop model, and most middle-aged and elderly people are not interested in this model. Although in the survey results, the popularity of Tea' stone cannot be compared with other beverage stores like tea, many people are trying this new model of pure tea drinks. Tea' stone's pure tea products are much higher than other beverage stores in terms of price. The survey results indicate that they are about $30 \%$ higher than the current fruit tea drinks. However, many people participating in the survey think that the product pricing is too expensive, and relatively few people are willing to try. 
The following figures are some statistical surveys of 100 young people randomly selected from nearby Tea' stone stores and friends. These people include milk tea lovers, pure tea lovers, non-tea lovers and people who don't care about it. Figure 1 is a survey on the brand awareness of Tea' stone. After introducing this new brand, how many of the 100 people are willing to try this new start-up pure tea drink:

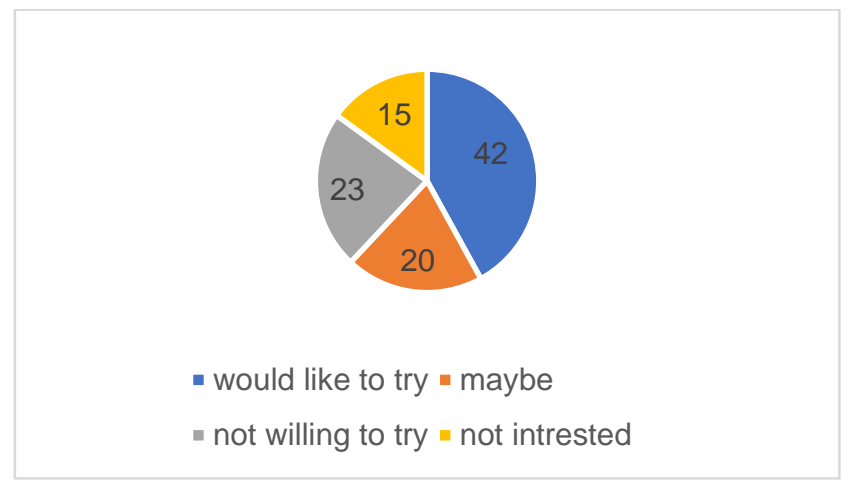

Figure 1. 100 people whether or not willing to try Tea' stone

As shown in figure 1, we can see that people still have a high acceptance of this new brand, which is mainly due to its innovative business philosophy, that is, to operate a pure tea beverage store in the mode of milk tea store. At the same time, Tea' stone's products are also attractive enough for many non-pure tea lovers to try. The next figure is the selection of similar tea brands on the market by different preference groups among the 100 people:

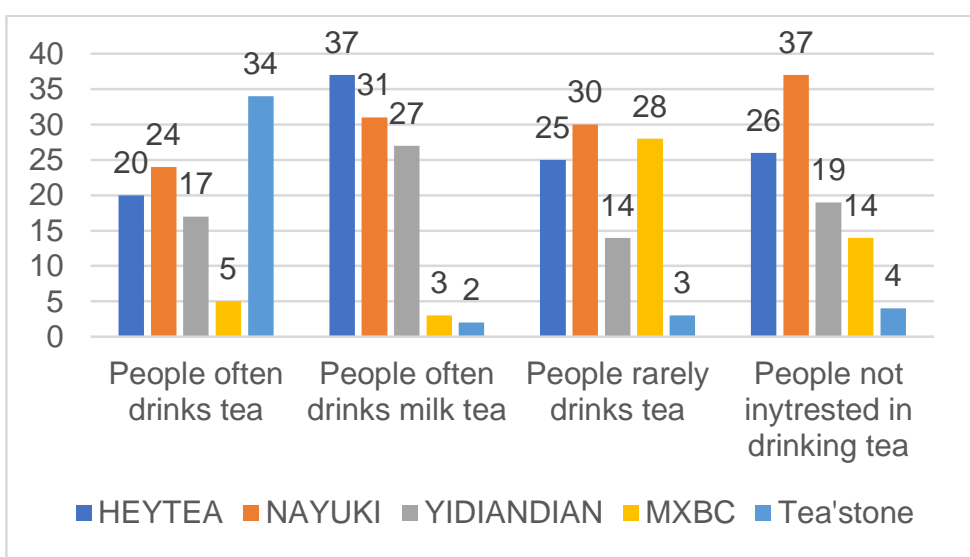

Figure 2. 100 people's choice of tea beverage brands with similar Tea' stone operating model in the market (percentage)

As shown in figure 2, we can clearly know that for young people who often drink tea, tea' stone's business philosophy and products are very attractive, and tea' stone accounts for the vast majority in this category. But, on the contrary, tea' stones may not be so attractive to those who don't drink much tea. I think this is because the brand only offers pure tea drinks. Figure 3 shows the views of people with different preferences on the price of tea' stone products:

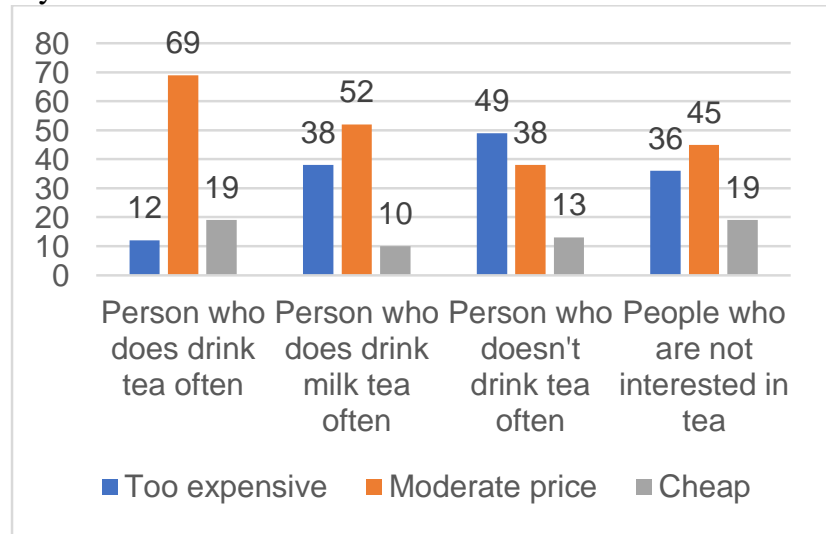

Figure 3. 100 people think tea' stone's products are too expensive or not (percentage) 
Accordingly, most tea lovers believe that the pricing of Tea' stone is reasonable because, based on the understanding of tea, pure tea drinks are inherently higher than other types of drinks. However, others who do not drink tea tend to think the price is on the high side. Lastly, figure 4 is a study whether these 100 people are optimistic about the brand Tea' stone, including whether they are optimistic about their development potential, whether they want more brands of this type to appear and whether they want more people to try this innovative pure tea drink:

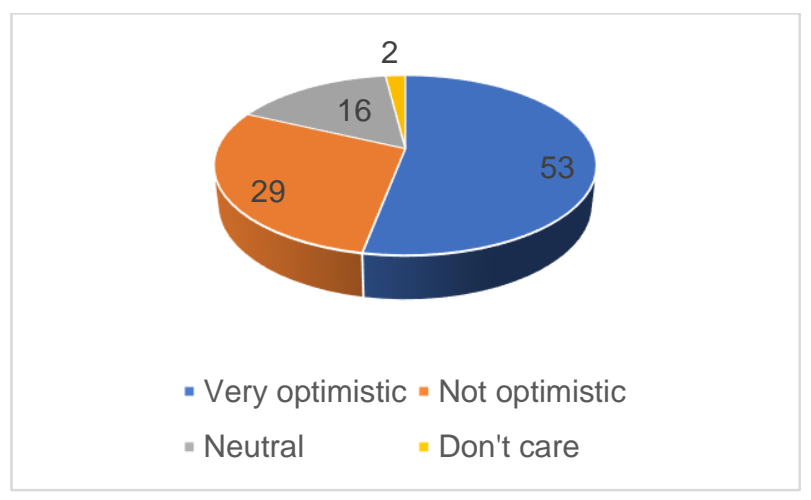

Figure 4. Whether these 100 people are optimistic about the brand Tea' stone

Generally speaking, most people are optimistic about tea' stone. They believe that this new innovative brand has many bright spots, such as an innovative business model and attractive products. However, opponents believe that tea' stone develops slowly, its products are priced too high, and the market is small (this is not the case). Neutral people believe that although Tea' stone has a lot of innovation, based on their ignorance of the market, they believe that tea' stone will face competitive pressure and development bottlenecks. Still, it has great development potential in the future.

\subsection{Context}

It is worth discussing these interesting facts revealed by the results of Zhangting's opinion. Compared with the wine industry, the current situation of the tea industry is that there are categories without brands, and the domestic market lacks domestic tea brands for mass consumers, especially young consumer groups. Tea' stone's goal is to become a new retail head brand of pure tea culture. In addition to the scene of drinking tea in the store, the store and online store applet mall also provide the same tea, characteristic tea ceremony, original tea utensils and tea life surrounding retail, and provide a full set of tea lifestyle solutions Online and offline [9].

With the rise of young consumers, the scale of new tea drinks has grown rapidly in recent years. Using the data of 2022-2027 China New Tea Industry Market Operation management and Investment Outlook Forecast Report, in 2019, China's new tea market scale has reached
97.8 billion yuan, up $83.15 \%$ year on year in 2018 . In 2020, China's new tea industry will enter the digital 3.0 stage. As a result, it is expected that China's new tea market size will exceed 100 billion yuan in 2020, reaching 102 billion yuan, and increase to 110.2 billion yuan in 2021[10].

Also, with the continuous expansion of new tea brands, the number of stores has continued to increase in recent years. As of the end of 2019, the number of newly made tea shops in my country reached 500,000. Affected by the epidemic in 2020, the number of new tea shops in China will decline, expected to be 480,000 . With the gradual control of the epidemic, the new tea industry has ushered in "compensatory consumption", and the number of stores will continue to increase in the future. From the perspective of store distribution, the offline stores of my country's new tea brands are mainly concentrated in firsttier cities such as Beijing, Shanghai, Guangzhou and Shenzhen. With the increasing demand of Chinese consumers for "healthy tea", the number of consumers of new-style tea drinks is increasing, reaching 305 million in 2019. In 2020, the number of consumers of new-style tea in my country was expected to exceed 340 million, and it will continue to increase in the future. From the perspective of the gender of consumers, women are the main consumers of new-style, ready-made tea drinks, and they are still in a dominant position, accounting for $60 \%$ of new-style tea consumers. Male consumers have increased significantly in recent years, accounting for $40 \%$ [11]

Judging from the age of the new-style tea consumer groups, young people are the main ones, among which the post-90s and post-00s have become the main consumer groups of new-style tea, accounting for about $70 \%$. Among post-90s and post-00s consumers, $28 \%$ of consumers who purchase new-style tea drinks have an average monthly consumption of more than 400 yuan, and $30 \%$ have an average monthly consumption between $200-400$ yuan. $26 \%$ between $100-200$ yuan [12].

\section{DISCUSSION}

The findings of the above analysis can be understood that Tea' stone, as an emerging start-up company, has many unique features. It has an experienced innovation management team and some necessary characteristics for a successful enterprise. It is very competitive in the market because it has a unique style and has no direct competitors. It has several advantages. The first advantage is they have a complete product chain. Their products, experience, packaging, scenes, and even furniture all come from the team's original design. They will be optimized and iterated in combination with the needs of the market and consumption scenes. Then they have abundant funds. They have Tens of millions of Yuan A round financing and tens of millions of angel round financing earlier this year. Thirdly, their market 
environment is good. There is no big competitor in their market. And the last one is their creative spirit. On this basis, combining the value of Chinese tea culture and combining the theory system of modern aesthetic design, they made innovative designs for every link of Chinese tea from drinking method, utensils and packaging to environment, experience, and marketing. It has a good mass base based on market research, and many young people will become potential customers of Tea' stone. This paper believes it can quickly occupy market share and gain a competitive advantage in the huge tea market. At the same time, several powerful investment companies have invested in Tea' stone, which shows that people favor this market and brand. At present, the only disadvantage of Tea' stone is its slow development, but its business model of physical stores causes this, but it is not enough to stop the development of tea' stone. It only needs time. This paper believes this is a company with the potential to become a leader in this field.

\section{CONCLUSION}

In conclusion, this paper analyzes the development potential and shortcomings of Tea' stone from many aspects and studies its advantages and disadvantages according to market research. This paper uses the POCD framework analysis method to comprehensively understand and compare the current problems, advantages and development potential of Tea' stone from three aspects: people, opportunity and context. The main conclusion that can be drawn from the POCD framework is that the company Tea' stone has a very experienced founding team, this can lead to potential success, also, because of the very innovative and attractive products, Tea' stone has the opportunity to seize the high ground in the tea market, coupled with the prosperity of the tea market in recent years, this is undoubtedly a good development opportunity for an emerging start-up tea company. In summary, this paper believes that Tea' stone is a company with great development potential and a start-up enterprise worthy of investors' attention.

\section{REFERENCES}

[1] Manish Chaulagain. Medium.com. POCD - a proven framework to build your new venture. May 9, 2020. Available at: https://medium.com/@manishchaulagain/pocd-aproven-framework-to-build-your-new-venture1 fc 3 ccaed016

[2] Manish Chaulagain. Medium.com. POCD - a proven framework to build your new venture. May 9, 2020. Available at: https://medium.com/@manishchaulagain/pocd-aproven-framework-to-build-your-new-venture$1 \mathrm{fc} 3 \mathrm{ccaed} 016$
[3] Zhaona. 21 Finance and Economics. Tea' stone, an innovative brand of pure tea, completed round a financing, and Mai Xing Investment and Zeng Ming jointly invested. March 31, 2021. Available at: https://m.21jingji.com/article/20210331/herald/74d 171f335dd1abbd2df57bbffab66a6.html

[4] Zhang Ting. 36Kr.com. 36Kr First Release | Pure Tea Innovation Brand "Tea' stone" Completes Series A Financing, Maixing Investment and Zeng Ming Joint Investment. March 31, 2021. Available at: https://www.36kr.com/p/1160513733592449

[5] Zhang Ting. 36Kr.com. 36Kr First Release | Pure Tea Innovation Brand "Tea' stone" Completes Series A Financing, Maixing Investment and Zeng Ming Joint Investment. March 31, 2021. Available at: https://www.36kr.com/p/1160513733592449

[6] Zhang Ting. 36Kr.com. 36Kr First Release | Pure Tea Innovation Brand "Tea' stone" Completes Series A Financing, Maixing Investment and Zeng Ming Joint Investment. March 31, 2021. Available at: https://www.36kr.com/p/1160513733592449

[7] Zhang Ting. 36Kr.com. 36Kr First Release | "Tea' stone" won tens of millions of yuan of angel round investment to locate a new retail brand of pure tea. January 25, 2021. Available at: https://www.36kr.com/p/1068377715074947

[8] Zhang Ting. 36Kr.com. 36Kr First Release | "Tea' stone" won tens of millions of yuan of angel round investment to locate a new retail brand of pure tea. January 25, 2021. Available at: https://www.36kr.com/p/1068377715074947

[9] Zhang Ting. 36Kr.com. 36Kr First Release | "Tea' stone" won tens of millions of yuan of angel round investment to locate a new retail brand of pure tea. January 25, 2021. Available at: https://www.36kr.com/p/1068377715074947

[10] Zhiyan Consulting Academy. Zhihu.com. In-depth research and development trend research report on China's ready-made tea market in 2021-2027. June 15, 2021. Available at: https://zhuanlan.zhihu.com/p/380680114

[11] Zhiyan Consulting Academy. Zhihu.com. In-depth research and development trend research report on China's ready-made tea market in 2021-2027. June 15, 2021. Available at: https://zhuanlan.zhihu.com/p/380680114

[12] Zhiyan Consulting Academy. Zhihu.com. In-depth research and development trend research report on China's ready-made tea market in 2021-2027. June 15, 2021. Available at: https://zhuanlan.zhihu.com/p/380680114 